\title{
Controle da intensidade do exercício aeróbio pela palpação da artéria radial
}

\author{
Aerobic exercise intensity can be controlled by palpation \\ of the radial artery
}

\author{
Anselmo José Perez \\ Kezia Donadia Dias ${ }^{1}$ \\ Luciana Carletti 1
}

1 Universidade Federal do Espírito Santo. Centro de Educação Física e Desportos. Vitória, ES, Brasil

Recebido em 27/04/09 Revisado em 10/08/09 Aprovado em 27/10/09
Resumo - A freqüência cardíaca (FC) durante o exercício tem sido utilizada no controle da intensidade de exercícios aeróbios. A palpação é o método mais popular e acessível para este fim. As respostas obtidas em testes laboratoriais são utilizadas para aplicações em campo, supondo-se que sejam semelhantes. $\mathrm{O}$ objetivo do estudo foi correlacionar as medidas de FC por palpação e por monitor de FC (MFC) em quatro sessões de exercício aeróbio e comparar os resultados obtidos entre sessões realizadas em laboratório e em campo. Participaram do estudo 15 mulheres jovens saudáveis que realizaram quatro sessões de exercício aeróbio, com carga contínua e duração de 40 minutos cada, sendo duas sessões em esteira e duas sessões em pista (50-60\% e 80-85\% FC max ). Durante as sessões, a FC foi registrada minuto a minuto pelo MFC (Polar Accurex) e medida a cada 10 minutos pela palpação do pulso radial. Foram observadas diferenças significativas entre a comparação das diferenças (deltas $-\Delta$ ) obtidas entre as medidas de FC pela palpação e pelo MFC, nas sessões de exercício na pista e no laboratório em apenas algumas situações. Foram observadas correlações fortes $(r=0,95)$ e moderadas para forte $(r=0,73)$ entre palpação e MFC nas quatro sessões. A FC de esforço na palpação foi subestimada não mais do que 5\%, mas não houve diferenças significativas entre os valores médios absolutos de FC mensuradas pelo MFC e pela palpação. Pode-se concluir que para uma mesma intensidade de esforço, os resultados obtidos no campo e laboratório foram semelhantes. Além disso, a FC pela palpação da artéria radial, imediatamente após o esforço, pode ser utilizada no controle de intensidade de exercícios aeróbios, representando uma monitorização fisiológica próxima da real obtida por MFC

Palavras-chave: Freqüência cardíaca; Exercício aeróbio; Monitorização fisiológica; Esforço físico; Palpação arterial.

Abstract - Heart rate (HR) during exercise has been used for the control of aerobic exercise intensity. Palpation is the most popular and accessible method for this purpose. The responses obtained in laboratory tests are used for field application and are suggested to be similar. The objective of this study was to correlate the HR measures obtained by palpation and with a heart rate monitor (HRM) in four sessions of aerobic exercise, and to compare the results obtained in the laboratory and in the field. Fifteen healthy young women underwent four sessions of aerobic training of 40 min each at a continuous load, including two sessions on a treadmill and two in the field (50-60\% and $80-85 \% \mathrm{HR}_{\text {max }}$ ). During the sessions, HR was recorded minute-by-minute with an HRM (Polar Accurex) and measured at 10-minute intervals by pulse palpation. There were significant differences in $\triangle H R$ values obtained by palpation and with the HRM between exercise sessions performed on the track and in the laboratory. Strong correlations $(r=0.95)$ and moderate to strong $(r=0.73)$ correlations were observed between palpation and HRM in the four sessions. HR during exercise obtained by palpation was underestimated by no more than 5\%, but there were no significant differences in mean absolute HR measured by HRM and palpation. For the same exercise intensity, similar results are obtained in the field and laboratory. In addition, HR obtained by palpation of the radial artery immediately after exercise can be used for the control of aerobic exercise intensity.

Key words: Heart rate; Aerobic exercise; Monitoring physiologic; Exertion; Arterial palpation. 


\section{INTRODUÇÃO}

A palpação das artérias radial e carótida para mensuração da frequência cardíaca (FC), além de ser uma técnica confiável ${ }^{1}$, ainda é o método de maior popularidade, por ser o mais acessível e apresentado nos principais livros textos de treinamento e fisiologia do exercício para monitorização do esforço físico. Além disso, com estreita relação entre o consumo de oxigênio $\left(\mathrm{VO}_{2}\right)$ e $\mathrm{FC}^{2,3}$, e relativa simplicidade de mensuração, a FC tem sido muito utilizada como parâmetro para prescrição e controle de intensidade de exercícios aeróbios ${ }^{4}$.

A mensuração da FC para o controle da intensidade do exercício pode ser obtida por diferentes métodos, como: eletrocardiograma (ECG), monitor de FC (MFC) e palpação arterial. O registro da atividade elétrica do coração pelo ECG é o método mais eficiente. Por outro lado, vários estudos têm demonstrado a boa relação existente entre MFC e ECG, tanto em repouso como em exercício ${ }^{5-8}$. Com o desenvolvimento tecnológico, várias marcas e modelos de MFC estão disponíveis, porém, nem sempre acessíveis economicamente para toda a população brasileira.

Apesar de Pollock et al. ${ }^{9}$ mostrarem que homens contavam com precisão as próprias FC por palpação, após o exercício, outros trabalhos como o de Bell e Bassey ${ }^{10}$ questionam essa validade, visto que o rápido declínio da $\mathrm{FC}$ após o exercício e o reduzido tempo em que deve ser contada a pulsação dificulta uma medida precisa.

Um estudo semelhante desenvolvido por Perez et al.? , a respeito da validade e confiabilidade do método de palpação da artéria radial para monitorização da FC em indivíduos do sexo masculino, evidenciou que esta técnica subestima o valor real da FC de esforço e de recuperação. Contudo, os autores sugerem que este método seja utilizado como controle de intensidade de treinamento, desde que interpretações cautelosas sejam realizadas, principalmente quanto à duração de tempo entre a retirada do estímulo e o início da contagem.

Por ser o método mais acessível, sejam em locais de prática de esportes, atividades ao ar livre, ou mesmo em academias, há necessidade de novas informações referentes à aplicabilidade prática da monitorização da FC pela palpação da artéria radial, no acompanhamento de diferentes intensidades de exercícios aeróbios.

Os objetivos deste trabalho foram: a) correlacionar os métodos de monitorização de FC por meio de MFC e por palpação da artéria radial em sessões de exercício aeróbio de diferentes intensidades (50$60 \%$ da FCmax e $80-85 \%$ da FCmax), realizadas em laboratório (esteira ergométrica) e em campo (pista de atletismo); b) comparar as diferenças obtidas (deltas $-\Delta$ ) entre as medidas por MFC e por palpação nas sessões de campo, com as diferenças obtidas (deltas $-\Delta$ ) entre as medidas por MFC e por palpação nas sessões laboratoriais de mesma intensidade de esforço.

\section{PROCEDIMENTOS METODOLÓGICOS}

\section{Amostra}

Não-probabilística, foi constituída por quinze indivíduos saudáveis, do sexo feminino, universitárias voluntárias ativas, após convite às turmas do curso de Educação Física da Universidade Federal do Espírito Santo (UFES), com idades entre 19 e 24 anos (21,5 \pm 0,4, média \pm EPM) e com o seguinte perfil antropométrico e de composição corporal: peso $56,8 \pm 2,0 \mathrm{~kg}$; estatura $165 \pm 1,0 \mathrm{~cm}$; IMC 20,7 $\pm 0,5$ $\mathrm{kg} / \mathrm{m}^{2}$; percentual de gordura $26,1 \pm 0,8 \%$, de acordo com métodos tradicionais de avaliação ${ }^{11}$.

A média da $\mathrm{FC}_{\text {max }}$ real obtida (195 $\left.\pm 2,0 \mathrm{bpm}\right)$ foi considerada próxima à esperada para a idade ${ }^{12}$ e $\mathrm{o}$ consumo máximo de oxigênio $\left(\mathrm{VO}_{2} \max 34,4 \pm 0,7\right.$ $\left.\mathrm{ml} \cdot \mathrm{kg}^{-1} \cdot \mathrm{min}^{-1}\right)$ entre fraco e regular para jovens sedentárias ${ }^{13}$.

Instrumentos para coleta de dados

Inicialmente, os sujeitos passaram por uma anamnese para diagnóstico clínico, com posteriores medidas de peso, estatura e dobras cutâneas, como rotina do Laboratório de Fisiologia do Exercício (LAFEX) do Centro de Educação Física e Desportos CEFD/ UFES. Os instrumentos utilizados foram: balança $(0,1 \mathrm{~kg})$ e toesa $(0,1 \mathrm{~m})$ da marca WELMY (São Paulo/SP, Brasil), além de um compasso da marca CESCORF (Porto Alegre/RS, Brasil) $(0,1 \mathrm{~mm})$.

Para a medida do $\mathrm{VO}_{2}$ max, de forma indireta, os sujeitos realizaram um teste ergométrico em esteira no LAFEX (INBRAMED, modelo KT 10200, Porto Alegre/RS, Brasil), pelo protocolo de Bruce $^{14}$. Durante o teste, a FC foi monitorada pelo sistema de registro eletrocardiográfico TEB - SM400 (Tecnologia Eletrônica Brasileira - São Paulo/SP, Brasil), com o objetivo de avaliação clínica ao esforço e identificação da FCmax. Os eletrodos foram colocados nas derivações MV5, D2M e V2M². Já durante as sessões de exercícios, em diferentes locais e intensidades, foi utilizado um MFC da marca Polar ${ }^{\circledR}$ (modelo Accurex Plus, Polar Electro Oy, FIN-90440 Kempele, Finland). 
Procedimento de coleta durante as sessões de exercício

Foram realizadas quatro sessões de exercício em dias diferentes, com intervalo mínimo de 24 h e máximo de $72 \mathrm{~h}$ entre uma sessão e outra. Duas sessões foram realizadas em esteira ergométrica no LAFEX (L1 e L2), e duas na pista de atletismo do CEFD/UFES (P1 e P2). A intensidade de esforço planejada foi de $50-60 \%$ da FCmax em L1 e P1, e de $80-85 \%$ da FCmax em L2 e P2. Os percentuais da intensidade de esforço foram calculados a partir da máxima FC atingida no teste ergométrico.

Cada sessão de exercício foi prescrita com duração de 40 minutos de esforço, mais três minutos de recuperação ativa. As sessões foram realizadas na sequência L1, P1, L2 e P2 e as temperaturas ambientes, nos dias de realização dos testes, variaram entre 24 e 27 graus.

O controle de L1 e L2 foi realizado via MFC e pela técnica de palpação da artéria radial. Na técnica de palpação, em razão do indivíduo estar com os dois braços estendidos lateralmente ao corpo, os batimentos foram contados pelos próprios avaliadores, em quinze segundos, e multiplicados por quatro, usando as pontas dos dedos indicador, médio e anular. Foram realizadas quatro interrupções do esforço, aos 10, 20, 30 e 40 minutos. A cada interrupção foram feitos, além dos registros de FC por MFC, duas mensurações de FC por palpação (PULSO A e PULSO B). Esse procedimento, em três etapas, foi assim realizado: etapa 1 - um avaliador (A1), no instante previamente determinado para a interrupção do esforço, acionava a parada da esteira e registrava a FC no MFC. Neste mesmo instante, antes da desaceleração total da esteira, ainda com a avaliada em movimento, outro avaliador (A2) iniciava a primeira mensuração da FC por palpação da artéria radial (PULSO A), no braço esquerdo da avaliada; etapa 2 - imediatamente após a parada total da esteira (momento em que a velocidade da esteira era igual a zero), o avaliador (A1) realizava a segunda medida da FC por palpação (PULSO B), no braço direito da avaliada; etapa 3 - no momento seguinte, era retomada a velocidade da esteira para o prosseguimento da atividade. O registro de PULSO A e de PULSO B permitiu comparar diferentes durações entre a parada do indivíduo e os valores mensurados de FC.

O controle de P1 e P2 foi realizado pelo MFC e pela técnica de palpação da artéria radial. A medida de FC pela palpação (PULSO) foi realizada pelas próprias voluntárias. Os batimentos cardíacos foram contados em quinze segundos, como em L1 e L2. Assim como no laboratório, para a realização das medidas de FC foram feitas quatro interrupções do esforço, aos 10, 20, 30 e 40 minutos. Este procedimento deu-se da seguinte maneira: após a medida da FC em repouso, o indivíduo iniciava a caminhada ou corrida, de acordo com a intensidade prescrita; nos momentos pré-determinados, o avaliador se aproximava do sujeito e, ao sinal do avaliador, o avaliado interrompia a atividade (parava), registrava a FC na memória do MFC e em seguida, realizava a mensuração da FC pela palpação, em quinze segundos, orientado pelo relógio do avaliador; terminada a medida, o sujeito informava o número de batimentos cardíacos contados ao avaliador e retomava a caminhada/corrida procurando manter a mesma intensidade de esforço, orientada pela marcação sonora de zona alvo do MFC.

Durante as mensurações, o relógio do MFC permanecia coberto por uma fita adesiva, para que nenhuma voluntária e/ou investigador tivesse conhecimento da FC registrada, antes que todas as medidas de FC por palpação da sessão correspondente fossem anotadas.

\section{Procedimentos estatísticos}

Para correlação entre as medidas de FC pelo MFC e pela palpação da artéria radial, utilizou-se o coeficiente de correlação de Pearson. Para comparação das diferenças (deltas $-\Delta$ ), obtidas entre as medidas de FC pela palpação e pelo MFC nas sessões de exercício, assim como para a comparação dos valores absolutos, utilizou-se ANOVA de uma via para medidas repetidas, com teste de comparação múltipla de Tukey. O teste t-Student para amostras pareadas foi utilizado para comparação entre as médias de MFC e palpação na pista. Foram considerados estatisticamente significativos os valores de probabilidade de $\mathrm{p}<0,05$. Para os cálculos estatísticos foi utilizado o programa Origin vs 3.5 (OriginLab Corporation, Northampton, USA).

\section{RESULTADOS}

Intensidade de esforço das sessões

Na Figura 1, observa-se a resposta de FC mensurada pelo MFC, minuto a minuto, nas quatro sessões de exercício aeróbio. A FC durante o decorrer do tempo foi mantida, em média, entre $112 \mathrm{bpm}$ e $123 \mathrm{bpm}$ para L1 e P1 (variação média de 11bpm) e entre $123 \mathrm{bpm}$ e $157 \mathrm{bpm}$ para L2 e P2 (variação média de $24 \mathrm{bpm}$ ). Isso representou percentuais médios de intensidade de esforço efetivamente realizadas de 63\% e 64\% da FCmax em L1 e P1, respectivamente, e $80 \%$ e $78 \%$ da FCmax para L2 e P2, respectivamente, apresentando características de equilíbrio 


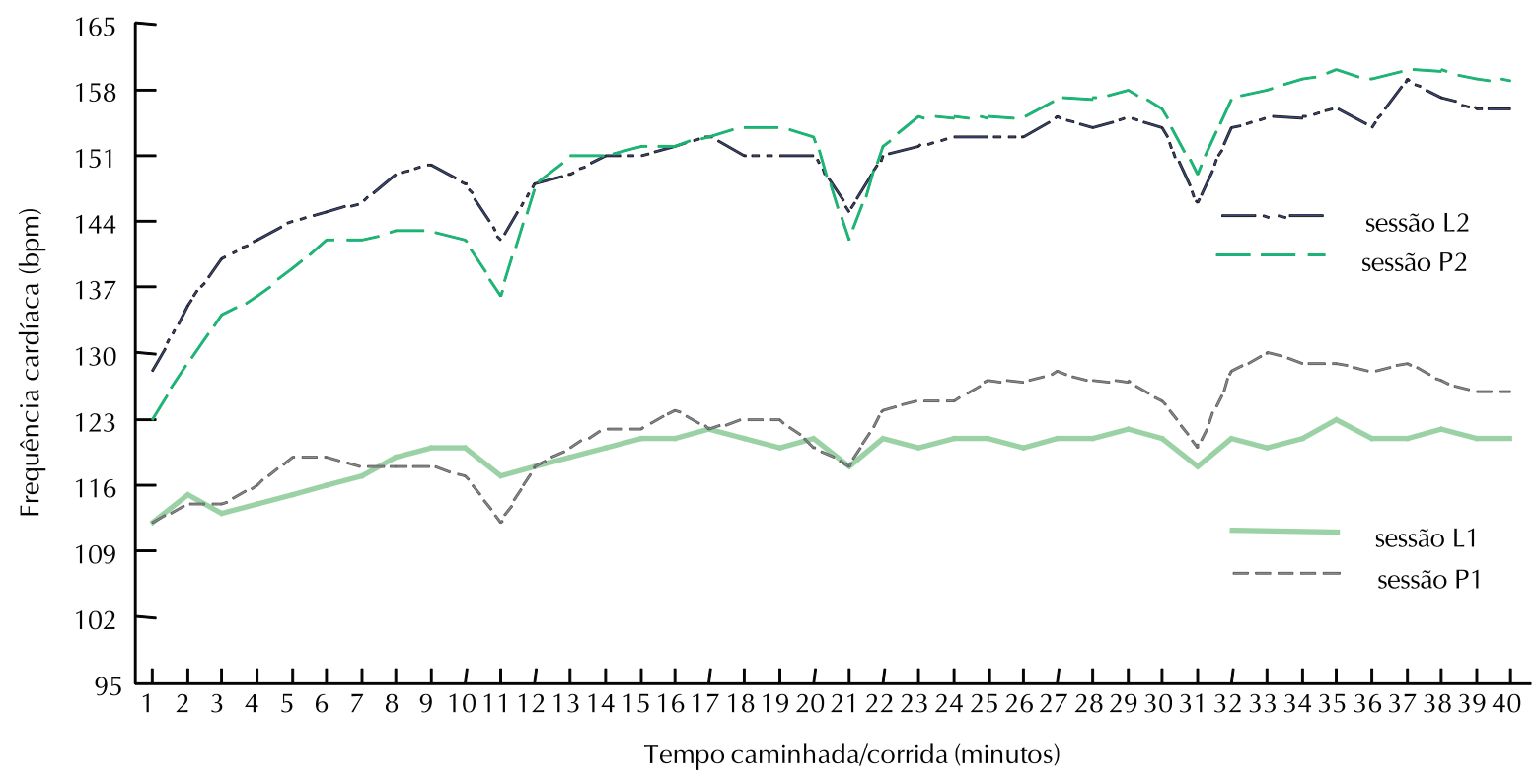

Figura 1. Respostas das FCs de universitárias ativas medidas por MFC durante 40 minutos, em quatro sessões de exercícios aeróbico; L1 - laboratório a 50-60\% da FCmax; L2 - laboratório a 50-85\% da FCmax; P1 - pista de atletismo a 50-60\% da FCmax; P2 - pista de atletismo a $80-85 \%$ da FCmax. $(n=15)$.

(steady state) provenientes de atividades do tipo aeróbias entre 60 e $90 \%$ da FCmax.

Houve diferença significativa entre as FCs quando a ANOVA de uma via foi aplicada entre as sessões de mesma intensidade apenas para L1 10min vs P1 40min, e P1 10min vs P1 40min. Já em L2 vs P2 as diferenças significativas foram para L2 $10 \mathrm{~min}$ vs L2 40min, L2 $10 \mathrm{~min}$ vs P2 30min e 40 min, L2 $20 \mathrm{~min}$ vs P2 10min e 40min, L2 30min vs P2 10min, L2 40min vs $\mathrm{P} 210 \mathrm{~min}, \mathrm{P} 210 \mathrm{~min}$ vs $\mathrm{P} 220 \mathrm{~min}, 30 \mathrm{~min}$ e $40 \mathrm{~min}$, e finalmente P2 20min vs P2 40min. de L1 e P1.

Nas comparações entre as diferentes intensidades, L1 foi significativamente diferente de L2, assim como P1 de P2.

Para uma visão de magnitude das FCs quanto às variações, se considerado do oitavo minuto de esforço em diante, a FC em L1 foi de 119bpm e no $40^{\circ}$ minuto, foi de $122 \mathrm{bpm}$, com variação de apenas três batimentos. As FCs observadas nessas sessões, tendo como referência o oitavo e o $40^{\circ}$ minuto, não apresentavam diferenças significantes, sendo: P1 118bpm e 126bpm (variação 8bpm); L2 - 149bpm e 156bpm (variação 7bpm); P2 - 143bpm e 159bpm (variação 16bpm). As quedas observadas nos valores de FC após 10, 20 e 30 minutos foram decorrentes das interrupções da atividade previamente determinadas para que as medidas de FC pela palpação fossem realizadas. Comportamentos semelhantes foram observados quando a mesma análise da Figura 1 foi feita para a palpação do pulso radial.
Monitorização da FC - MFC e palpação da artéria radial - Sessões realizadas em laboratório (L1 e L2)

O tempo total utilizado na primeira medida de FC pela palpação (PULSO A) foi, em média, de 19 segundos, sendo 4 segundos para localização da pulsação e mais 15 segundos para a contagem. Na segunda medida pela palpação (PULSO B), o tempo total utilizado foi de 30 segundos, em média, 15 segundos para a desaceleração total da esteira e 15 segundos para a contagem dos batimentos.

Na Tabela 1, observam-se correlações altas e significativas entre o MFC e PULSO A, e entre MFC e PULSO B. As diferenças entre os valores absolutos das medidas pelo MFC e pela palpação, variando entre $0,8 \%$ e $5 \%$ para $\mathrm{L} 1$, e entre $2,0 \%$ e 3,4\% para L2, foram sempre maiores entre MFC e PULSO B.

\section{Sessões realizadas em campo (P1 e P2)}

$\mathrm{Na}$ Tabela 2, são apresentados os valores de FC mensurados pelo MFC e pela técnica de palpação nos momentos pré-determinados. Assim como no laboratório, foram encontradas correlações altas e significativas. As maiores diferenças observadas entre os valores absolutos de FC em P1 foram: 5,3\%, na recuperação; $3,9 \%$ na última medida de esforço. Já em P2, encontrou-se 6\% na recuperação e, durante o esforço, 3\% nos 20 minutos de atividade. 
Tabela 1. Medidas de FC pelo MFC e pela palpação da artéria radial, realizadas nas sessões L1 e L2 em repouso, esforço e recuperação $(n=15)$

\begin{tabular}{|c|c|c|c|c|c|}
\hline L 1 & MFC (bpm) & PULSO A (bpm) & PULSO B (bpm) & r MFC/PULSO A & r MFC/PULSO B \\
\hline Repouso & $80 \pm 2$ & - & $79 \pm 3$ & - & 0,93 \\
\hline $10 \mathrm{~min}$ & $120 \pm 3$ & $117 \pm 3$ & $114 \pm 3$ & 0,88 & 0,83 \\
\hline $20 \mathrm{~min}$ & $121 \pm 3$ & $119 \pm 3$ & $116 \pm 3$ & 0,92 & 0,83 \\
\hline $30 \mathrm{~min}$ & $121 \pm 3$ & $120 \pm 3$ & $117 \pm 3$ & 0,88 & 0,87 \\
\hline 40min & $122 \pm 3$ & $120 \pm 3$ & $118 \pm 3$ & 0,88 & 0,88 \\
\hline $\operatorname{Rec} 3 \min$ & $103 \pm 3$ & $105 \pm 3$ & $101 \pm 2$ & 0,79 & 0,91 \\
\hline \multicolumn{6}{|l|}{ L 2} \\
\hline Repouso & $81 \pm 2$ & - & $81 \pm 2$ & - & 0,97 \\
\hline $10 \mathrm{~min}$ & $148 \pm 4$ & $145 \pm 5$ & $143 \pm 4$ & 0,85 & 0,90 \\
\hline $20 \mathrm{~min}$ & $151 \pm 4$ & $150 \pm 4$ & $147 \pm 4$ & 0,99 & 0,90 \\
\hline $30 \mathrm{~min}$ & $154 \pm 4$ & $155 \pm 4$ & $149 \pm 4$ & 0,94 & 0,91 \\
\hline $40 \mathrm{~min}$ & $156 \pm 4$ & $157 \pm 5$ & $152 \pm 5$ & 0,92 & 0,90 \\
\hline Rec 3min & $117 \pm 3$ & $117 \pm 4$ & $115 \pm 4$ & 0,95 & 0,98 \\
\hline
\end{tabular}

L1 - sessão de exercício realizada no laboratório com intensidade de 50-60\% da FCmax; L2 - sessão de exercício realizada no laboratório com intensidade de $80-85 \%$ da FCmax; MFC - medida da FC por monitor de FC; PULSO A - medida de FC por palpação da artéria radial (batimentos contados em 15 segundos), realizada antes da desaceleração total da esteira; PULSO B - medida de FC por palpação da artéria radial (batimentos contados em 15 segundos), realizada após a parada total da esteira; 10, 20, 30 e 40 minutos - momentos de interrupção da atividade para realização das medidas de FC; Rec 3min. - 3o minuto de recuperação; Valores expressos em médias EEPM. Todos os valores de correlação (r) foram significativos com p<0,05. Não houve diferenças entre as médias quando ANOVA de uma via e teste de Tukey foram aplicados.

Tabela 2. Medidas de FC pelo MFC e pela palpação da artéria radial realizadas na sessão P1 e P2 em repouso, esforço e em recuperação $(n=15)$

\begin{tabular}{lccc}
\hline P1 & MFC (bpm) & PULSO (bpm) & $r$ \\
\hline Repouso & $88 \pm 2$ & $88 \pm 2$ & 0,97 \\
10min & $119 \pm 2$ & $117 \pm 3$ & 0,89 \\
20min & $122 \pm 2$ & $121 \pm 3$ & 0,73 \\
30min & $126 \pm 3$ & $123 \pm 3$ & 0,88 \\
\hline 40min & $128 \pm 2$ & $123 \pm 2$ & 0,79 \\
Rec 3min & $114 \pm 3$ & $108 \pm 3$ & 0,75 \\
\hline P2 & & & \\
Repouso & $89 \pm 2$ & $89 \pm 3$ & 0,89 \\
10min & $142 \pm 4$ & $143 \pm 5$ & 0,95 \\
20min & $152 \pm 4$ & $147 \pm 5$ & 0,57 \\
\hline 30min & $156 \pm 4$ & $154 \pm 5$ & 0,92 \\
40min & $159 \pm 5$ & $159 \pm 4$ & 0,93 \\
Rec 3min & $133 \pm 6$ & $125 \pm 4$ & 0,83 \\
\hline
\end{tabular}

P1 - sessão de exercício realizada em pista de atletismo com intensidade de $50-60 \%$ da $\mathrm{FC}_{\max } ;$ P 2 - sessão de exercício realizada em pista de atletismo com intensidade de $80-85 \%$ da $\mathrm{FC}_{\max } ; \mathrm{MFC}$ - medida da FC por monitor de FC; PULSO - medida de FC por palpação da artéria radial (batimentos contados em 15 segundos); 10, 20, 30 e 40 minutos- momentos de interrupção da atividade para realização das medidas de FC; Rec 3 min - $3^{\circ}$ minuto de recuperação; Valores expressos em médias \pm EPM. Todos os valores de correlação foram significativos com $p<0,05$. Não houve diferenças significativas entre as médias quanto teste t-Student para amostras pareadas foi aplicado.

\section{Diferenças (deltas - $\Delta$ ) obtidas entre MFC e palpação da artéria radial:}

As comparações entre as diferenças $(\Delta)$ dos valores de FC medidos pelo MFC e pela palpação, aos 10,
20, 30 e 40 minutos, em P1, L1A e L1B, estão apresentadas na Figura 2. Houve diferença significativa $(\mathrm{p}<0,05)$ somente entre P1 10min $(-3 \mathrm{bpm})$ vs L1B $10 \mathrm{~min}(-10 \mathrm{bpm})$, e entre L1B $10 \mathrm{~min}$ e as medidas nos tempos seguintes, exceto com L1B aos 20, 30 e $40 \mathrm{~min}$. Para uma noção de magnitude, nota-se que o maior delta negativo foi de -10bpm em L1B 10min, enquanto que L1A $30 \mathrm{~min}$ foi o menor $(-3 \mathrm{bpm})$. Em P1, o maior delta negativo foi de $-3 \mathrm{bpm}$ aos 10 minutos de esforço. Houve diferença significativa $(\mathrm{p}<0,05)$ apenas entre P1 10min vs L1B 10min.

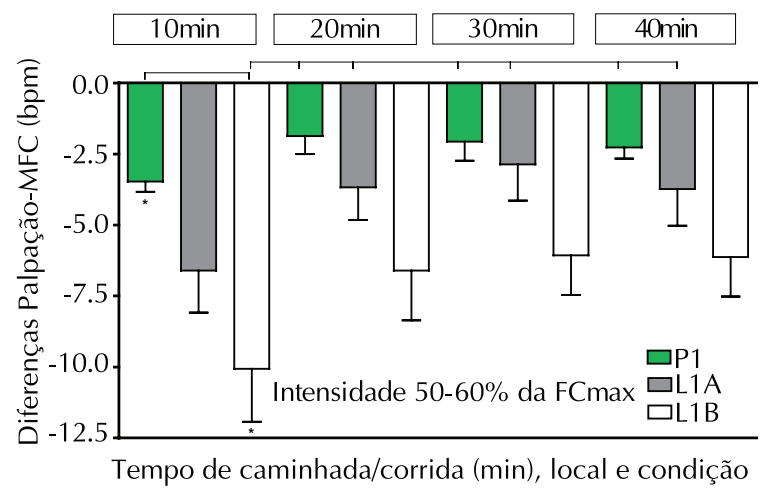

Figura 2. Comparação das diferenças (delta $-\Delta$ ) entre as médias dos valores de FC mensuradas aos 10, 20, 30 e 40 minutos de exercício, pela papação (PULSO) e pelo MFC, nas sessões P1, L1A e L1B, realizadas em pistas e em laboratórios, respectivamente. ANOVA de uma via, com teste de Tukey. * $\mathrm{p}<0,05$ Dados apresentados por média \pm EPM.

As comparações entre os deltas de FC medidos pelo MFC e pela palpação, aos 10, 20, 30 e 40 minutos, em L2 e P2 estão apresentadas na Figura 3. Houve diferença 
significativa somente de L2B 10min vs L2A 30min e $40 \mathrm{~min}$. Nas sessões com intensidade mais forte, todos os deltas foram negativos e em L2A ocorreram menores valores do que em $\mathrm{L} 2 \mathrm{~B}$, mas não significativos. $\mathrm{Os}$ menores deltas negativos foram de $-5 \mathrm{bpm}$ na pista $(\mathrm{P} 1$ 10min), e de -10bpm em laboratório (L2B 10min).

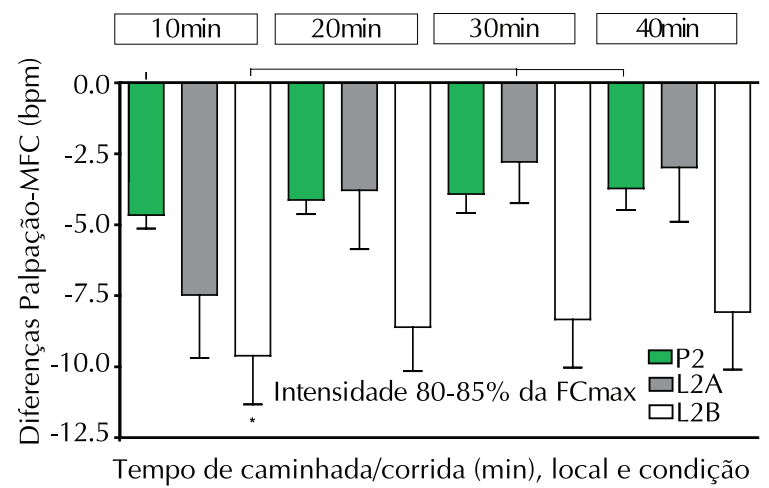

Figura 3. Comparação das diferenças (delta $-\Delta$ ) entre as médias dos valores de FC mensuradas aos 10, 20, 30 e 40 minutos de exercício, pela papação (PULSO) e pelo MFC, nas sessões P2, L2A e L2B, realizadas em pistas e em laboratórios, respectivamente. ANOVA de uma via, com teste de Tukey. $*$ p $<0,05$ Dados apresentados por média \pm EPM.

Além dos resultados apresentados nas Figuras 2 e 3 , poucas diferenças significativas foram encontradas entre os deltas das sessões fracas e fortes, $50-60 \%$ da FCmax e $80-85 \%$ da FCmax, respectivamente. Foram: L1B 10 min vs L2A 30min, L2A 40min e L2B 40min; P1 20min vs L2B 10min e L2B 20min; P1 30min vs L2B 10 min; L1A 30min vs $\mathrm{L} 2 \mathrm{~B} 10 \mathrm{~min}$; $\mathrm{P} 140 \mathrm{~min}$ vs $\mathrm{L} 2 \mathrm{~B} 10 \mathrm{~min}$.

\section{DISCUSSÃO}

De acordo com o objetivo deste trabalho, utilizar a FC medida por meio da palpação da artéria radial para identificar a intensidade utilizada no exercício aeróbio mostrou-se uma estratégia viável, independente da intensidade.

Intensidade de esforço das sessões

Podemos afirmar que os percentuais de intensidade de esforço planejados para este trabalho foram atingidos e que as sessões se caracterizaram como aeróbias, com efetivo equilíbrio entre demanda e consumo de oxigênio (steady-state), como apresentado na Figura 1. Vários fatores podem afetar o desempenho observado no campo e no laboratório, entre eles destacam-se: temperatura ambiente, umidade do ar e adaptabilidade aos equipamentos utilizados, mesmo mantidas as especificidades do exercício ${ }^{15}$.

Os percentuais médios de intensidade de esforço atingidos em L1 e P1 não apresentaram diferenças significativas, assim como os percentuais atingidos em L2 e P2, com exceção de alguns momentos. Kenny et $\mathrm{al}^{15}$ demonstraram que, em intensidades mais elevadas, próximas do $\mathrm{VO}_{2}$ max, as diferenças entre campo e laboratório diminuem ou até desaparecem, e isso pode ser evidenciado neste trabalho.

Apesar dos cuidados em elaborar um protocolo de laboratório o mais próximo possível do modo e condições da sessão executada em campo ${ }^{16}$, as elevações observadas nos valores de FC durante o tempo dos 40min em L1 e em P1 (Figura 1), podem ser explicadas pela influência de fatores ambientais, não controlados no campo. Mesmo assim, isso não descaracterizou a estabilidade da FC durante o exercício, o que reforça a utilização da estratégia de palpação em situação real de caminhada ou corrida.

As sessões de intensidades mais altas (L2 e P2) apresentaram diferenças significativas apenas nos sete minutos iniciais da atividade (dados não apresentados), possivelmente pela dificuldade das avaliadas em iniciar a atividade no campo com uma FC mais elevada, correspondente a uma intensidade de $80 \%$ da FCmax atingida e mantida voluntariamente. No laboratório, esta intensidade era imposta pela velocidade da esteira. Verifica-se, também, em P2, a influência dos fatores externos, tendo em vista a elevação da FC ocorrida durante essa sessão, entre o oitavo minuto e quadragésimo, que foi de $16 \mathrm{bpm}$, em relação ao aumento de apenas 7 bpm ocorrido na sessão L2.

A FC é regulada pela influência simpática e parassimpática do sistema nervoso autônomo. Para intensidades baixas de esforço, assim como no início do exercício físico (até aproximadamente $100 \mathrm{bpm}$ ), o aumento da FC é atribuído, principalmente, à retirada da estimulação parassimpática ${ }^{17-19}$, que não só aumenta a contratilidade dos átrios, mas também eleva a velocidade de condução da onda de despolarização dos ventrículos a partir do nódulo $\mathrm{AV}$, independentemente do nível de intensidade do esforço e do nível de condicionamento aeróbio de indivíduos saudáveis ${ }^{20}$.

Segundo Rowell ${ }^{19}$, para intensidades de esforço baixas até, aproximadamente, $40 \%$ do $\mathrm{VO}_{2} \max$, a FC se eleva rapidamente em função da retirada da influência parassimpática. Já em intensidades acima de $40 \%$ do $\mathrm{VO}_{2}$ max, a ativação simpática se torna dominante e a resposta da FC, consequentemente, torna-se mais lenta.

A FC em L1 manteve-se mais constante que nas demais sessões, correspondendo à fase de steady state. Durante exercícios moderados, o steady state é alcançado em, aproximadamente, 3 minutos, para 
indivíduos normais ${ }^{6}$. Nas sessões de campo (P1 e P2), a maior dificuldade na manutenção desse equilíbrio, possivelmente, possa ser explicada pela influência de fatores externos, como já citado, e, nas sessões de intensidade mais elevadas (L2 e P2), por maior custo metabólico. Como afirmam Ferrero e Vaquero ${ }^{25}$, o steady state se alcança com maior ou menor rapidez em função da cinética do mecanismo de consumo de oxigênio, diretamente relacionado a $\mathrm{FC}^{21}$.

MFC e Palpação da artéria radial:

Como observado na Figura 2, às magnitudes dos deltas negativos encontrados nas mensurações feitas na pista não passaram de -3bpm e eram menores, apesar de não serem significativamente diferentes, do que em relação aos deltas das mensurações no laboratório. Em intensidade baixa de esforço aeróbio, mesmo com a parada total do sujeito para a tomada e contagem do pulso, a técnica de palpação subestima de forma não significativa a FC de esforço.

No laboratório, demonstrou-se que o tempo total utilizado para fazer a primeira mensuração de FC pela palpação - PULSO A - foi menor que o tempo total utilizado para fazer a segunda medida - PULSO B - aproximadamente, 19 e 30 segundos, respectivamente, incluindo os $15 \mathrm{~s}$ de contagem do pulso. A observação de que as diferenças entre PULSO A e MFC foram sempre menores que as diferenças entre PULSO B e MFC está de acordo com Pollock et al. ${ }^{9}$, que observaram maiores erros quando a medida de FC por palpação era realizada em tempo superior a 15 s, imediatamente após o exercício. Além do tempo máximo de demora, deve-se ter cautela na contagem dos batimentos em tempos inferiores a este, pois quanto menor for o tempo de contagem, maior será o erro quando a conta não for precisa ${ }^{22}$. Um único erro na contagem do pulso pode resultar em erros de 4, 6, 10 ou $12 \mathrm{bpm}$ no valor da FC, dependendo do tempo utilizado na contagem, ou seja, 15, 10, 6 ou 5 segundos, respectivamente ${ }^{11}$. Alguns autores têm incluído a discussão sobre o tempo de duração da mensuração da FC pelo pulso e parece haver consenso de que existe semelhança nos valores encontrados em 10 ou 15 segundos $^{23-26}$. O Colégio Americano de Medicina Esportiva ${ }^{1}$ aconselha durante o exercício contar por $15 \mathrm{~s}$, mas sugere uma contagem de pulso de 6 s ou 10s em função da rápida diminuição após o exercício. Dentro dessas variações, pelos dados encontrados em nosso trabalho e como descrito na literatura, pode ser indicado o tempo de 10s para a mensuração da FC durante o exercício.

É importante destacar, ainda, que a medida do PULSO A era realizada sem a interrupção total do esforço, enquanto que a medida do PULSO B ocorreu depois de parada completa da esteira. Os maiores valores de frequência cardíaca obtidos no momento da medida do PULSO A podem ser resultantes da manutenção da resposta de retroalimentação dos mecanorreceptores musculares para ativação do componente simpático do sistema nervoso autôno$\mathrm{mo}^{33}$. Ressalta-se que em P1 e P2, as medidas de FC pela palpação foram realizadas pelas próprias avaliadas, que eram instruídas sem criar-se expectativa ou excessiva ansiedade para o ato de mensuração.

McArdle et al..$^{28}$ ao compararem a FC de exercício e de recuperação, observaram que a FC após o exercício não era um bom indicador da intensidade de esforço. Considerando cargas absolutas de traba1ho, eles observaram que quando a medida de FC por palpação era realizada em 14 segundos, a queda da FC seria de 5,7\% em exercício intenso (180bpm) e de $13,5 \%$ para cargas de trabalho mais moderadas (140bpm). No presente estudo, as quedas de FC observadas entre MFC e palpação tiveram percentuais menores. No laboratório (L1 e L2), as maiores diferenças verificadas entre MFC e PULSO A, durante o esforço, foram de $2,5 \%$ na sessão de intensidade mais leve e de 2,0\% na sessão de intensidade mais forte. Entre MFC e PULSO B, essas diferenças foram de $5 \%$ e 3,4\%, em L1 e L2, respectivamente. Na pista, as maiores diferenças de FC observadas entre MFCe palpação durante o esforço foram de 3,9\% na sessão mais leve e de 3,0\% na sessão mais forte. Estes resultados estão próximos aos observados por Pollock et al. ${ }^{9}$ que verificaram uma queda de menos de $2 \%$ na FC dentro de 12-14 segundos após a interrupção do exercício, para uma intensidade de $80 \%$ da FCmax. Assim, quanto mais intensa for a atividade, mais lenta será a queda da FC na interrupção do esforço, possivelmente, pelo maior tempo necessário para a retirada da estimulação simpática ${ }^{19}$.

De acordo com os estudos desenvolvidos por Bell e Bassey ${ }^{10}$, a maior fonte de erro na técnica de palpação é atribuída à habilidade individual em contar os batimentos com precisão. Ainda considerando esta situação, Pollock et al. ${ }^{9}$ observaram que, no uso desta técnica, os erros dos avaliadores e dos avaliados podem ser resultados de tempo insuficiente de prática da técnica. Perez et al. ${ }^{7}$ observaram que a FC mensurada pela palpação subestimava a FC medida pelo MFC entre 5 e $15 \%$, mas se mantendo dentro de faixas de zona alvo utilizadas em programas de treinamento aeróbio. 
No presente estudo, os resultados confirmam a boa relação entre a FC medida pela palpação, imediatamente após o esforço e a FC registrada pelo MFC ao final do exercício. Os valores de FC medidos pela palpação da artéria radial, tanto nas sessões de intensidades mais leves (L1 e P1), como nas sessões de intensidades mais fortes (L2 e P2), apresentaram correlações de moderada a forte $(\mathrm{r}$ $=0,75)$, assim como correlações fortes $(r=0,95)$. Apesar da boa relação, a medida por palpação pode apresentar valores menores que os registrados no MFC, não sendo, contudo, significativos. Desta forma, essa técnica de medida da FC pela palpação pode ser empregada em programas de treinamento aeróbio dentro da zona de frequência ou zona alvo ${ }^{2,28-30}$. Dentro dessa zona alvo, estímulos entre 60 e $90 \%$ da FCmax seriam considerados como adequados para produzir efeitos agudos e crônicos positivos ao sistema cardiovascular ${ }^{2}$.

\section{CONCLUSÃO}

A medida da FC pela técnica de palpação da artéria radial, desde que mensurada até, no máximo, trinta segundos após a parada da corrida, subestima de forma não significativa os valores de FC obtidos por MFC, durante ou ao final de uma sessão de exercício, entre $1 \%$ a $5 \%$, o que representa uma intensidade dentro das faixas de estímulo cardiovascular, podendo, assim, ser utilizada no controle de intensidade de exercícios aeróbios. Além disso, para uma mesma intensidade de esforço, os resultados obtidos no campo são semelhantes aos resultados obtidos no laboratório.

\section{REFERÊNCIAS BIBLIOGRÁFICAS}

1. Colégio Americano de Medicina Esportiva (ACSM). Teste de esforço e prescrição de exercício. Rio de Janeiro: Revinter; 2007.

2. Gilman MB. The use of heart rate to monitor the intensity of endurance training. Sports Medicine 1996;21(2):73-79.

3. Swain DP, Abernathy KS, Smith CS, Lee SJ, Bunn SA. Targert heart rates for the development of cardiorespiratory fitness. Med Sci Sports Exerc 1994;26(1):112-116.

4. Almeida MB. Frequência cardíaca e exercício: uma interpretação baseada em evidências. Rev Bras Cineantropom Desempenho Hum 2007;9(2)196-202.

5. Léger L, Thivierge M. Heart rate monitors: validity, stability, and functionality. Phys Sportsmed 1988;16(5):143-151.

6. Louro LPS. Validade da freqüência cardíaca obtida pelo pulso radial no controle de intensidade de treinamento aeróbio. [Monografia de Licenciatura Plena]. Vitória (ES): Universidade Federal do Espírito Santo; 1999.
7. Perez AJ, Louro LPS, Dias KD, Magalhães MA. Validade da técnica de palpação da freqüência cardíaca pelo pulso radial no controle da intensidade de sessões de treinamento aeróbio (Resumo). Anais do XXII Simpósio Internacional de Ciências do Esporte. São Paulo: 1999. p.80.

8. Kingsley M, Lewis MJ, Marson RE. Comparison of Polar 810s and an ambulatory ECG system for RR interval measurement during progressive exercise. Int J Sports Med 2005;26:39-44.

9. Pollock ML, Broida J, Kendrick Z. Validity of the palpation technique of heart rate determination and its estimation of training heart rate. Res Q 1972;43(1):77-81.

10. Bell JM, Bassey EJ. Postexercise heart rates and pulse palpation as a means of determining exercising intensity in an aerobic dance class. Br J Sports Med 1996;30:48-52.

11. De Rose HE, Pigatto E, De Rose RFC. Cineantropometria, educação física e treinamento desportivo. Rio de Janeiro: FAE, SEED; 1984.

12. Guedes DP. Composição corporal: princípios, técnicas e aplicações. Londrina: Associação dos Professores de Educação Física; 1994.

13. Karvonen MJ, Kentala E, Mustala O. The effects of training on heart rate: a "longitudinal" study. Ann Med Exp Biol Fenn. 1957;35(3):307-315

14. Bruce RA, Kusumi FE, Hosmer D. Maximal oxygen intake and nomographic assessment of functional aerobic impairment in cardiovascular disease. Am Heart J 1973;85(4):546-562.

15. Kenny GP, Reardon FD, Marion A, Thoden JS. A comparative analysis of physiological responses at submaximal workloads during different laboratory simulations of field cycling. Eur J Appl Physiol 1995;71(5):409-415.

16. Wilmore, JH. The assessment of and variation in aerobic power in world class athletes as related to specific sports. Am J Sports Med 1984;12(2):120-127.

17. Nóbrega ACL, Araújo CGS. Heart rate transient at the onset of active and passive dynamic exercise. Med Sci Sports Exerc 1993;25(1):37-41.

18. Rowell LB. Human cardiovascular control. New York: Oxford University Press; 1993. p.162-203.

19. Rowell LB, O'Leary DS. Reflex control of the circulation during exercise: chemoreflexes and mechanoreflexes. J Appl Physiol 1990;69(2):407-418.

20. Almeida MB, Araújo CGS. Efeitos do treinamento aeróbico sobre a freqüência cardíaca. Rev Bras Med Esporte 2003;9(2):104-112.

21. Strath SJ, Swartz AM, Bassett JR DR, O'Brien WL, King GA, AInsworth BE. Evaluation of heart rate as a method for assessing moderate intensity physical activity. Med Sci Sports Exerc 2000;32(9), Suppl., S465-S470.

22. Edwards S. Heart zone training: exercise smart, stay fit, and live longer. Massachusets: Adams Media Corporation; 1996.

23. Oldridge NB, Haskell WL, Single P. Carotid palpation, coronary heart disease and exercise rehabilitation. Med Sci Sports Exerc 1981;13(1):6-8.

24. Boone T, Rape SM. Carotid palpation and heart rate changes in postmyocardial infarction patients. Arch Phys Med Rehabil 1983;64(11):543-547. 
25. Boone T, Frentz KL, Boyd NR Carotid palpation at two exercise intensities. Med Sci Sports Exerc 1985;17(6):705-709.

26. DeVan AE, Lacy BK, Cortez-Cooper MY, Tanaka H. Post-exercise palpation of pulse rates: its applicability to habitual exercisers. Scand J Med Sci Sports 2005;15(3):177-181.

27. Brum PC, Forjaz CLM, Tinucci T, Negrão CE. Adaptações agudas e crônicas do exercício físico no sistema cardiovascular. Rev Paul Edu Fís 2004;18(N. esp.):21-31.

28. McArdle WD, Zwiren L, Magel JR. Validity of the postexercise heart rate as a means of estimating heart rate during work of varying intensities. Res $Q$ 1969;40(3):523-528.

29. Lambert MI, Mbambo ZH, Gibson STC. Heart rate during training and competition for long-distance running. J Sports Sci 1998;16(3):S85-S90.
30. Karvonen J, Vuorimaa T. Heart rate and exercise intensity during sports activities: pratical application. Sports Med 1988;5(5):303-311.

\section{Endereço para correspondência}

Anselmo José Perez

Av. Fernando Ferrari, s/n - Laboratório de Fisiologia do Exercício

Centro de Educação Física e Desportos

Universidade Federal do Espírito Santo. Goiabeiras CEP - 29060-900 - Vitória. ES

E-mail: anselmo@cefd.ufes.br 